\title{
Anti-Müllerian hormone serum level and other markers associated with pregnancy outcome in oocyte donation
}

\author{
Anne-Sophie Delesalle ${ }^{1}$, Geoffroy Robin ${ }^{1}$, Patricia Thomas-Desrousseaux ${ }^{1}$, Didier Dewailly ${ }^{1,2}$ \\ and Sophie Catteau-Jonard ${ }^{1,2^{*}}$
}

\begin{abstract}
Background: Oocyte donation is a medical technique used principally for woman with ovarian failure. Optimizing donor recruitment is essential to obtain the best results with this technique. Understanding how donor parameters influence outcome for the recipients is fundamental. The aim of this study was to determine whether clinical and/ or biological parameters in the donors influence the chance of pregnancy in recipients. Our objective was also to verify whether the outcomes of controlled ovarian stimulation (COS) are predictive of pregnancy in the recipients.

Methods: A retrospective observational study was conducted in the Department of Reproductive Medicine in the Lille University Hospital. Between September 2005 and April 2014, COS was performed in 145 donors for 308 recipients' cycles. We compared the cycles whose outcome was pregnancy to the cycles without pregnancy. Quantitative variables were compared using the nonparametric Mann-Whitney test. Qualitative variables were compared using a Chi-2 test or Fisher exact test, according to the numbers. Covariance analysis was performed to adjust for potential confounding factors.

Results: The donors who produced at least one pregnancy had a mean baseline serum anti-Müllerian hormone $(\mathrm{AMH})$ level significantly higher than those who did not $(p=0.001)$. The mean antral follicle count did not differ between the 2 groups. After covariance analysis controlling for the number of couples attributed to a given donor, this difference remained significant $(p=0.029)$. Mature follicle number, estradiol serum level at the trigger day, number of mature oocytes and embryo number were significantly higher in the donors who produced pregnancy.
\end{abstract}

Conclusion: Serum AMH level is associated with pregnancy outcome after oocyte donation.

Keywords: Anti-Müllerian hormone, Oocyte donation, Donors, Pregnancy outcome, Antral follicle count

\section{Background}

Oocyte donation is a medical technique used principally for women with ovarian failure. At the end of 2011 in France, 1,806 couples were waiting for oocyte donation, whereas only 402 couples received a donation the same year. Recently, this activity increased slightly but insufficiently to address all requests, resulting in a long delay for recipient couples. Optimizing donor recruitment is essential to obtain the best results with this technique.

\footnotetext{
* Correspondence: sophie.jonard@chru-lille.fr

${ }^{1}$ Departments of Endocrine Gynaecology and Reproductive Medicine, Hôpital Jeanne de Flandre, C.H.R.U., Faculty of Medicine, Université de Lille II, F-59000 Lille, France

${ }^{2}$ INSERM U1172 Team 2, JPARC, Université de Lille II, F-59000 Lille, France
}

Understanding how donor characteristics influence outcome of the recipients is fundamental. Many clinical or biological parameters can influence ART results such as the woman's age, ovarian reserve and oocyte quality. Anti-Müllerian hormone (AMH) is an interesting biological marker because it reflects ovarian reserve. It can also predict ovarian response to controlled ovarian stimulation (COS). However the value of this biological marker to predict pregnancy in oocyte donation is unknown.

The aim of this study was to determine whether clinical and/or biological parameters in the donor influence the chance of pregnancy in recipients. We also verified whether the COS outcomes are predictive of pregnancy 
in the recipients. For this, we compared cycles resulting in pregnancy with cycles which did not result in pregnancy.

\section{Methods}

All oocyte donation cycles performed in the Department of Reproductive Medicine in the Lille University Hospital between September 2005 and April 2014 were analyzed retrospectively. Oocyte donation cycles were performed in accordance with the bioethics law. This study was approved by the institutional Review Board of Lille University Hospital. All patients gave their informed consent before oocyte donation.

\section{Population}

All potential oocyte donors underwent consultation with a referring physician of the Department. They were under the age of 38 years and had at least one child. About $25 \%$ of the donors were altruistic. The other donors were relatives (i.e., recruited by recipient couple) but we performed anonymous cross-donations.

Donors with hereditary pathology, abnormal karyotype, body mass index $(\mathrm{BMI})>35 \mathrm{~kg} / \mathrm{m} 2, \mathrm{AMH}<1.5 \mathrm{ng} / \mathrm{mL}$, $\mathrm{AFC}<10$ and/or abnormal serology were rejected. The donors underwent a rigorous clinical evaluation in search of contraindication to oocyte donation, including hereditary pathology. On the day of the consultation, ovarian reserve was evaluated by serum AMH assay (complemented by serum FSH and estradiol assays between days 2 and 5 of a spontaneous cycle), and antral follicle count (AFC, sum of both ovaries) by transvaginal ultrasonography (Voluson E8 Expert, GE Healthcare). Karyotype, psychological evaluation and HIV1-2, HTLV, HBV, HCV, syphilis, and CMV serology completed the evaluation.

Serum AMH levels were assessed using the second generation enzyme immunoassay AMH-EIA (ref. A16507) provided by Beckman Coulter Immunotech (Villepinte, France).

For matching donors and recipients, phenotypic characteristics (geographic origin, color of skin, eyes and hair, weight, height) and blood group were registered. A donor was allocated to one, two or three recipient(s) according to her ovarian reserve and the number of oocytes at the puncture.

\section{Donor cycle}

All donors COS was performed in the department of Reproductive Medicine in University Hospital. One cycle was performed by one donor for one, two or three recipient's couples.

The recipients received endometrial preparation synchronously to the donor stimulation using hormonal replacement treatment, and GnRH agonists if the recipient still cycled. Recipients were started on oral micronized estradiol (6 mg /day). Evaluation of endometrial thickness was performed at D14 and considered satisfactory if $\geq 7 \mathrm{~mm}$. Vaginal micronized progesterone $(800 \mathrm{mg} /$ day $)$ was initiated on the evening of donor oocyte retrieval.

A long agonist protocol was used for donors' stimulation between September 2005 and December 2011. From January 2012, an antagonist protocol was used. COS was performed with gonadotropins, individually adjusted based on AFC, baseline serum AMH level, age and body mass index (BMI) values, and adapted during stimulation according to ultrasound findings and estradiol levels. When there were at least 2 follicles measuring $>18 \mathrm{~mm}$ mean diameter, ovulation was triggered either by subcutaneous recombinant hCG $0.25 \mathrm{mg}$ (Ovitrel*; Merck Serono) or by GnRH agonist (triptoreline) $0.2 \mathrm{mg}$ (Decapeptyl; Ipsen Pharma) in case of antagonist protocol. Follicles were aspirated transvaginally with ultrasound guidance 35 to $36 \mathrm{~h}$ thereafter. Fertilization was performed by intracytoplasmic sperm injection (ICSI) with the sperm of the spouse. Embryo transfer of 1 or 2 embryos was performed at days 2 or 3 . If the embryo quality was sufficient, surplus embryos were frozen. The transfer of frozen embryos was performed under hormonal replacement protocol (oral estrogen and intravaginal progesterone) identical to the fresh transfer. Fourteen days after the embryo transfer, serum $\beta$-HCG level was measured. Transvaginal ultrasonography was performed at 5-6 weeks gestation to assess the presence of a gestational sac.

\section{Data collection}

Data on age, BMI, parity, smoking, serum AMH levels and AFC were collected.

For each donor cycle, total dose of gonadotropins, duration of stimulation, number of follicles $>15 \mathrm{~mm}$ and estradiol serum level the day of trigger and the number of collected oocytes were recorded.

Clinical pregnancy was defined by the presence of an intrauterine gestational sac with heart activity on ultrasound performed at 5-6 weeks of amenorrhea.

Birth was defined as delivery of a child after at least 24 weeks of amenorrhea.

\section{Statistical analysis}

Statistical analyses were performed using the Statistical Package for Social Sciences (SPSS 22.0 for Windows). Quantitative variables were compared using the nonparametric Mann-Whitney test. Qualitative variables were compared using a Chi-2 test or Fisher exact test, according to the numbers. Covariance analysis was performed to adjust for potential confounding factors.

\section{Results}

Between September 2005 and April 2014, 145 donors underwent $\mathrm{COS}$ in our department for 308 recipients. 
Table 1 Clinical, biological and ultrasound characteristics of oocyte donors

\begin{tabular}{|c|c|}
\hline Age (years) ${ }^{a}$ & $32(23-37)^{a}$ \\
\hline Child number $^{a}$ & $2(1-4)^{a}$ \\
\hline BMI $\left(\mathrm{kg} / \mathrm{m}^{2}\right)^{\mathrm{a}}$ & $23(18,5-31)^{a}$ \\
\hline $\mathrm{AMH}(\mathrm{ng} / \mathrm{mL})^{\mathrm{a}}$ & $3.9(1,8-9.7)^{\mathrm{a}}$ \\
\hline Antral follicle court (sum of both ovaries) ${ }^{a}$ & $20(10-42.5]^{a}$ \\
\hline Smoker ${ }^{\mathrm{b}}$ & $28 \%$ \\
\hline Overweight $\left\{\mathrm{BM} \geq 25 \mathrm{~kg} / \mathrm{m}^{2}\right)^{\mathrm{b}}$ & $38 \%$ \\
\hline
\end{tabular}

${ }^{a}$ Median [5th percentile-55th percentile]

bercent

One donor had COS cancelled because of ovarian hyperstimulation syndrome and 2 because of absence of ovarian response. For seven donors, we performed no embryo transfer due to poor oocyte or embryo quality. Over a period of 8 years, 418 transfer cycles were performed: 288 with fresh embryos (69 \%) and 130 with frozen embryos (31\%).

Clinical, biological and ultrasound characteristics of the donors are summarized in Table $1.28 \%$ of donors were overweight or obese $\left(\mathrm{BMI}=25-35 \mathrm{~kg} / \mathrm{m}^{2}\right)$ and $38 \%$ were smokers.

The outcomes of the 145 donors COS are shown in Table 2. The donors whose eggs resulted in at least one pregnancy $(\mathrm{n}=85)$ had serum AMH level significantly higher than those who did not $(\mathrm{n}=60) \quad(p=0.001)$ (Table 3). After covariance analysis controlling for the number of couples attributed to a donor, the difference remained significant $(p=0.029)$. However, receiver operating characteristic (ROC) curve analysis showed that for pregnancy outcome, donors' AMH values yielded a low area under the curve (0.665) [CI: 057-0.76], insufficient to reach a predictive threshold. For example, a threshold at $5 \mathrm{ng} / \mathrm{mL}$ gave a specificity of $75 \%$ but a sensitivity of only $41 \%$. Concerning the stimulation parameters, mean mature follicle number, serum estradiol level at the trigger day, number of mature oocytes and embryo number were significantly higher in the donors who achieved pregnancy. The mean total dose of used

Table 2 Outcomes of the of the 145 donor's COS

\begin{tabular}{ll}
\hline & Mean (SD) \\
\hline Number of mature follicles & $14.5( \pm 7)$ \\
Duration of stimulation (days) & $12.2( \pm 1.7)$ \\
Estradiol on the trigger day $(\mathrm{pg} / \mathrm{mL})$ & $2128( \pm 1142)$ \\
Total dose of gonadotropins $(\mathrm{UI})$ & $2926( \pm 955)$ \\
Number of mature oocytes & $9.6( \pm 6)$ \\
Number of embryos & $6( \pm 4.1)$ \\
Pregnancies rate per donor & $90 \%$ \\
Birth rate per donor & $70 \%$ \\
\hline
\end{tabular}

Table 3 Comparisons between the donors achieving pregnancy and the others

\begin{tabular}{|c|c|c|c|}
\hline & Pregnancy & No pregnancy & $P$ \\
\hline Number of oocyte donors & 85 & 60 & \\
\hline Age (years) ${ }^{a}$ & $31[23-37]$ & 32 [24.9-37] & 0.425 \\
\hline $\mathrm{BMI} \geq 25$ & $27.1 \%$ & $45.7 \%$ & 0.064 \\
\hline Smokers & $37.3 \%$ & $37.5 \%$ & 0,897 \\
\hline $\begin{array}{l}\text { Number of recipients } \\
\text { per donor }{ }^{\mathrm{a}}\end{array}$ & $3[1-3]$ & $2[1-4]$ & 0,001 \\
\hline $\begin{array}{l}\text { Child number of the } \\
\text { donor }^{\mathrm{a}}\end{array}$ & $2[1-4]$ & $1.9[1-3.55]$ & 0.096 \\
\hline $\mathrm{AMH}(\mathrm{ng} / \mathrm{mL})^{\mathrm{a}}$ & $4,48[2.23-9.96]$ & $3.15[1.65-8.6]$ & 0,001 \\
\hline $\begin{array}{l}\text { Antral follicle count } \\
\text { (sum of both ovaries) }\end{array}$ & 20 [11-49] & 19 [10-36] & 0.095 \\
\hline Number of mature follicles ${ }^{b}$ & $16.6 \pm 7.4$ & $11.5 \pm 5.2$ & $<0.001$ \\
\hline Duration of stimulation (days) & $12.1 \pm 1.7$ & $12.4 \pm 1,8$ & 0.26 \\
\hline $\begin{array}{l}\text { Estradiol on the trigger } \\
\text { day }(\mathrm{pg} / \mathrm{mL})^{\mathrm{b}}\end{array}$ & $2429 \pm 1136$ & $1694 \pm 10.1$ & $<0.001$ \\
\hline $\begin{array}{l}\text { Total dose of } \\
\text { gonadotropins }(U I)^{b}\end{array}$ & $2650 \pm 856$ & $3315 \pm 959$ & $<0.001$ \\
\hline Number of mature oocytes ${ }^{b}$ & $11.3 \pm 6.1$ & $7.2 \pm 4.9$ & $<0.001$ \\
\hline Number of embryos ${ }^{b}$ & $7.5 \pm 4.2$ & $4 \pm 3.2$ & $<0.001$ \\
\hline
\end{tabular}

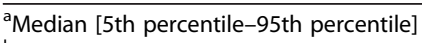

${ }^{\mathrm{b}}$ Mean \pm standard deviation

gonadotropins was higher in donors without pregnancy. Conversely, stimulation duration did not differ significantly between the two groups (Table 3 ).

The results of recipient cycles are summarized in Table 4. After the 145 donors' COS, 1,380 mature oocytes were given to 308 recipients (mean \pm SD: $4.5 \pm 1.7$ oocytes per recipient and per cycle). After fertilization by ICSI, 870 embryos were obtained, i.e. $2.9 \pm 1.7$ embryos per recipient. Among the embryos, $77 \%$ were transferred.

Table 4 Results of recipient cycles

\begin{tabular}{ll}
\hline & Value \\
\hline Number of recipient cycles & 308 \\
Number of transfers & 418 \\
Number of pregnancies & 133 \\
Number of miscarriages & 42 \\
Number of births & 108 \\
Number of oocytes per recipient per cycle & \\
Number of transferred embryos per cycle & \\
Implantation rate & $4,48 \pm 1.68$ \\
Pregnancy rate per transfer & $1.59 \pm 0.51$ \\
Pregnancy rate per cycle (fresh and thawed embryos) & $23 \%$ \\
Birth rate per transfer & $31.8 \%$ \\
Miscarriage rate & $43.2 \%$ \\
Birth rate per cycle (fresh and thawed embryos) & $25.1 \%$ \\
aMean \pm standard deviation & $31.6 \%$
\end{tabular}

${ }^{\mathrm{a}}$ Mean \pm standard deviation 
Among these, $73 \%$ were transferred immediately and $27 \%$ after thawing. A mean of 2.3 embryos were transferred to recipient per donor COS (immediately and after thawing), with an average of 1.6 embryos per transfer.

\section{Discussion}

The aim of this study was to identify whether features in the oocyte donors were associated with pregnancy in the recipient.

We did not observe an influence of donor's age on pregnancy outcome, probably because most of the donors were young. Indeed, only $13.1 \%$ of our donors were over 35 years old. In the literature, most of studies did not observe influence of donor's age on oocyte donation outcome [1-4]. The use of ovarian reserve markers, such as serum AMH, to select the donors with a good potential ovarian response lead to refusal of older donors who more frequently have an AMH serum level lower than $1.5 \mathrm{ng} / \mathrm{mL}$. Therefore, the age effect on pregnancy outcome is less important than in the setting of IVF in infertile women using their own oocytes.

We found a trend for a negative influence of donor's BMI on pregnancy outcome. Overweight and obesity are responsible for impaired oocyte quality [5]. However, our results were not statistically significant and published data are controversial. We excluded donors having a BMI higher than $35 \mathrm{~kg} / \mathrm{m}^{2}$, because of the difficulties and the risks linked to COS and puncture.

The high predictive value of serum AMH level on response to COS has already been reported [6, 7]. Our study was in agreement with other series of donors showing a good correlation between serum AMH level and ovarian response: number of collected oocytes, total dose of gonadotropins used and serum estradiol level the day of trigger [8-14]. Thus, serum AMH level is a reliable marker to obtain the largest number of oocytes while trying to limit the occurrence of ovarian hyperstimulation syndrome [8-12]. The minimum threshold of $\mathrm{AMH}$ at $1.5 \mathrm{ng} / \mathrm{mL}$, that we used in our center, has been reported to provide a sensitivity of $86 \%$ and a specificity of $78 \%$ to predict a sufficient ovarian response [8].

In our study, the AFC was not different between the donors who achieved a pregnancy and those who did not, although serum AMH level was higher in the former. To our knowledge, this is the first study reporting a significantly higher AMH level in donors that produced a pregnancy compared with those who did not. Moreover, this difference persisted after adjustment for the number of recipients assigned to each donor, which would be a bias via the increased number of transfers. Previously, several authors have shown that serum AMH level was associated with oocyte quality, that could explain the association of AMH with pregnancy occurrence
[15-19]. A recent meta-analysis reported a strong association between serum AMH level and the occurrence of live birth after in vitro fertilization, but the predictive value of AMH was very low [20]. Similarly in our study, we were not able to identify an AMH threshold value that could predict with sufficient power the outcome of oocyte donation. Indeed, the area under the ROC curve was low (AUC $=0.665$; IC: $0.57-0.76$ ). This is confirmed by five other studies $[8,9,11,13,14]$. For example, Riggs et al. found an AUC at 0.559 [8]. Thus, serum AMH level is associated with the outcome of an oocyte donation, but its predictive value is poor.

\section{Conclusion}

Our study updates the importance of AMH to evaluate ovarian reserve of oocyte donors. AMH was shown to be correlated to the donor's response to COS and to the number of oocytes allocated to one or more recipients. Although serum AMH level is associated with pregnancy outcome after oocyte donation, its predictive value is insufficient to be used to select donors by itself.

\section{Abbreviations \\ AFC: antral follicle count; AMH: anti-Müllerian hormone; ART: assisted reproductive technology; BMI: body mass index; COS: controlled ovarian hyperstimulation; ICSI: intracytoplasmic sperm injection; ROC: receiver operating characteristic.}

\section{Competing interests}

The authors declare that they have no competing interests.

\section{Authors' contributions}

ASD: study design, data collection and analysis, manuscript writing and revision; GR: data collection and manuscript revision; PTD: data collection, expert knowledge and manuscript revision; DD: study design, data analysis, manuscript revision and expert knowledge; SCJ: study design and coordination, data analysis, manuscript revision and expert knowledge. All authors read and approved the final manuscript.

\section{Acknowledgments}

The authors thank Dr. Marcelle Cedars (University of California, San Francisco, USA) for assistance in language corrections.

\section{Funding}

None declared.

Received: 7 October 2015 Accepted: 12 January 2016

Published online: 19 January 2016

\section{References}

1. Barton SE, Missmer SA, Ashby RK, Ginsburg ES. Multivariate analysis of the association between oocyte donor characteristics, including basal follicle stimulating hormone (FSH) and age, and IVF cycle outcomes. Fertil Steril. 2010;94:1292-5

2. Shulman A, Frenkel Y, Dor J, Levran D, Shiff E, Maschiach S. The best donor. Hum Reprod. 1999;14:2493-6.

3. Wong IL, Legro RS, Lindheim SR, Paulson RJ, Sauer MV. Efficacy of oocytes donated by older women in an oocyte donation programme. Hum Reprod. 1996;11:820-3.

4. Harris SE, Faddy M, Levett S, Sharma V, Gosden R. Analysis of donor heterogeneity as a factor affecting the clinical outcome of oocyte donation. Hum Fertil Camb Engl. 2002;5:193-8.

5. Pantasri T, Norman RJ. The effects of being overweight and obese on female reproduction: a review. Gynecol Endocrinol. 2014;30:90-4. 
6. La Marca A, Sighinolfi G, Radi D, Argento C, Baraldi E, Artenisio AC, et al. Anti-Mullerian hormone (AMH) as a predictive marker in assisted reproductive technology (ART). Hum Reprod Update. 2010;16:113-30.

7. Niodromiti S, Anderson RA, Nelson SM. Technical and performance characteristics of anti-Müllerian hormone and antral follicle count as biomarkers of ovarian response. Hum Reprod. 2014;21(6):698-710.

8. Riggs R, Kimble T, Oehninger S, Bocca S, Zhao Y, Leader B, et al. Anti-Müllerian hormone serum levels predict response to controlled ovarian hyperstimulation but not embryo quality or pregnancy outcome in oocyte donation. Fertil Steril. 2011;95:410-2.

9. Bukulmez O, Li Q, Carr BR, Leader B, Doody KM, Doody KJ. Repetitive oocyte donation does not decrease serum anti-Müllerian hormone levels. Fertil Steril. 2010;94:905-12.

10. Nakhuda GS, Douglas NC, Thornton MH, Guarnaccia MM, Lobo R, Sauer MV. Anti-Müllerian hormone testing is useful for individualization of stimulation protocols in oocyte donors. Reprod Biomed Online. 2010;20:42-7.

11. Talebian S, Licciardi F, Liu M, Grifo JA, Krey LC. Assessing anti-mullerian hormone (AMH) as a marker of ovarian response in anonymous oocyte donors: quantity or quality? Fertil Steril. 2008;90:5267.

12. Hazout A, Cohen Bacrie P, Mendoza R, Mendoza C, Tesarik J. Antimullerian Hormone, Ovarian Reserve and Oocyte Quality in Young Oocyte Donors. Fertil Steril. 2005:84:S251.

13. Polyzos NP, Stoop D, Blockeel C, Adriaensen P, Platteau P, Anckaert E, et al. Anti-Müllerian hormone for the assessment of ovarian response in GnRH-antagonist-treated oocyte donors. Reprod Biomed Online. 2012;24:532-9.

14. Martínez F, Clua E, Carreras O, Tur R, Rodríguez I, Barri PN. Is AMH useful to reduce low ovarian response to $\mathrm{GnRH}$ antagonist protocol in oocyte donors? Gynecol Endocrinol. 2013;29:754-7.

15. Ebner T, Sommergruber M, Moser M, Shebl O, Schreier-Lechner E, Tews G. Basal level of anti-Müllerian hormone is associated with oocyte quality in stimulated cycles. Hum Reprod. 2006;21:2022-6.

16. Majumder K, Gelbaya TA, Laing I, Nardo LG. The use of anti-Müllerian hormone and antral follicle count to predict the potential of oocytes and embryos. Eur J Obstet Gynecol Reprod Biol. 2010;150:166-70.

17. Irez T, Ocal P, Guralp O, Cetin M, Aydogan B, Sahmay S. Different serum anti-Müllerian hormone concentrations are associated with oocyte quality, embryo development parameters and IVF-ICSI outcomes. Arch Gynecol Obstet. 2011:284:1295-301.

18. Brodin T, Hadziosmanovic N, Berglund L, Olovsson M, Holte J. Antimüllerian hormone levels are strongly associated with live-birth rates after assisted reproduction. J Clin Endocrinol Metab. 2013;98:1107-14.

19. Lin W-Q, Yao L-N, Zhang D-X, Zhang W, Yang X-J, Yu R. The predictive value of anti-Mullerian hormone on embryo quality, blastocyst development, and pregnancy rate following in vitro fertilization-embryo transfer (IVF-ET). J Assist Reprod Genet. 2013;30:649-55.

20. Iliodromiti S, Kelsey TW, Wu O, Anderson RA, Nelson SM. The predictive accuracy of anti-Müllerian hormone for live birth after assisted conception: a systematic review and meta-analysis of the literature. Hum Reprod Update. 2014:20:560-70.

\section{Submit your next manuscript to BioMed Central and we will help you at every step:}

- We accept pre-submission inquiries

- Our selector tool helps you to find the most relevant journal

- We provide round the clock customer support

- Convenient online submission

- Thorough peer review

- Inclusion in PubMed and all major indexing services

- Maximum visibility for your research

Submit your manuscript at www.biomedcentral.com/submit

) Biomed Central 